\title{
Generalizations of Cauchy-Schwarz inequality in unitary spaces
}

Leng Tuo*

"Correspondence:
tleng@shu.edu.cn
School of Computer Engineering
and Science, Shanghai University,
Shanghai, China

\begin{abstract}
In this paper, we give a generalization of Cauchy-Schwarz inequality in unitary spaces and obtain its integral analogs. As an application, we establish an inequality for covariances.
\end{abstract}

MSC: $26 \mathrm{D} 15 ; 62 \mathrm{~J} 10$

Keywords: Cauchy-Schwarz inequality; unitary space; positive measure

\section{Introduction}

Let $u$ and $v$ be two vectors in a unitary space $\mathbb{H}$. The Cauchy-Schwarz inequality is well known,

$$
|\langle u, v\rangle| \leq\|u\| \cdot\|v\|,
$$

where $\langle\cdot, \cdot\rangle$ and $\|\cdot\|$ denote the inner product and norm in $\mathbb{H}$, respectively. Its integral form in the space of real-valued functions $L^{2}[a, b]$ is

$$
\left(\int_{a}^{b} f \cdot g d \mu\right)^{2} \leq\left(\int_{a}^{b} f^{2} d \mu\right)\left(\int_{a}^{b} g^{2} d \mu\right) .
$$

The Cauchy-Schwarz inequality is one of the most important inequalities in mathematics. To date, a large number of generalizations and refinements of the inequalities (1.1) and (1.2) have been investigated in the literature (see [1] and references therein, also see [2-9]).

In this note, we will present some new generalizations of the Cauchy-Schwarz inequality (1.1).

Suppose that $\mathbb{H}$ is a unitary space (complex inner product space) with standard inner product $\langle\cdot, \cdot\rangle$ and norm $\|\cdot\|$, namely $\langle x, y\rangle=x^{T} \bar{y}$ and $\|x\|=\sqrt{\langle x, x\rangle}$ (see [10]). Let $X=\left(x_{1}, x_{2}, \ldots, x_{n}\right)$ denote the $n$-tuple of vectors $x_{i} \in \mathbb{H}, i=1, \ldots, n$. For two $n$-tuples $X=\left(x_{1}, \ldots, x_{n}\right)$ and $Y=\left(y_{1}, \ldots, y_{n}\right)$ of $\mathbb{H}$, we define this $A$-product of vector $x_{i}$ and $y_{i}$ for $X$ and $Y$ by

$$
x_{i} \otimes_{A} y_{i}=\left\langle x_{i}, y_{i}\right\rangle-\left\langle x_{i}, b\right\rangle-\left\langle a, y_{i}\right\rangle
$$

where $a=\frac{x_{1}+\cdots+x_{n}}{n}$ and $b=\frac{y_{1}+\cdots+y_{n}}{n}$.

Our main results are the following theorems.

(c) 2015 Tuo. This article is distributed under the terms of the Creative Commons Attribution 4.0 International License (http://creativecommons.org/licenses/by/4.0/), which permits unrestricted use, distribution, and reproduction in any medium, provided you give appropriate credit to the original author(s) and the source, provide a link to the Creative Commons license, and indicate if changes were made. 
Theorem 1 Let $X=\left(x_{1}, \ldots, x_{n}\right)$ and $Y=\left(y_{1}, \ldots, y_{n}\right)$ be two $n$-tuples of the unitary space $\mathbb{H}$, then

$$
\sum_{i=1}^{n}\left|x_{i} \otimes_{A} y_{i}\right| \leq\left(\sum_{i=1}^{n}\left\|x_{i}\right\|^{2}\right)^{1 / 2}\left(\sum_{i=1}^{n}\left\|y_{i}\right\|^{2}\right)^{1 / 2}
$$

Equality holds if $y_{i}=\left(x_{i}-2 a\right) \lambda(i=1, \ldots, n)$ for any $\left\{x_{1}, \ldots, x_{n}\right\}$, where $\lambda$ is a non-negative constant.

In particular, if $n=1$, then (1.3) is the Cauchy-Schwarz inequality (1.1).

For complex numbers $\mathbb{C}$, by Theorem 1 , we have the following.

Corollary 1 Suppose that $x_{1}, \ldots, x_{n}$ and $y_{1}, \ldots, y_{n}$ are complex numbers. Set

$$
a=\frac{x_{1}+\cdots+x_{n}}{n}, \quad b=\frac{y_{1}+\cdots+y_{n}}{n},
$$

then

$$
\left(\sum_{i=1}^{n}\left|x_{i} y_{i}-a y_{i}-b x_{i}\right|\right)^{2} \leq\left(\sum_{i=1}^{n}\left|x_{i}\right|^{2}\right)\left(\sum_{i=}^{n}\left|y_{i}\right|^{2}\right) .
$$

Equality holds if $y_{i}=\left(x_{i}-2 a\right) \lambda(i=1, \ldots, n)$ for any $\left\{x_{1}, \ldots, x_{n}\right\}$, where $\lambda$ is a non-negative constant.

Let $H \oplus H \oplus \cdots \oplus H$ denote the direct sum of $n$ unitary space $\mathbb{H}$ with norm $\|X\|=$ $\left(\sum_{i=1}^{n}\left\|x_{i}\right\|^{2}\right)^{\frac{1}{2}}$. Set $f(X, Y)=\sum_{i=1}^{n} x_{i} \otimes_{A} y_{i}$. Since $f(X, X)$ is not always non-negative, $f(X, Y)$ is not an inner product in the above direct sum. Hence, (1.3) is different from the CauchySchwarz inequality in the above direct sum.

If we set $\left|X \otimes_{A} Y\right|=\sum_{i=1}^{n}\left|x_{i} \otimes_{A} y_{i}\right|$, then (1.3) can be restated as

$\left|X \otimes_{A} Y\right| \leq\|X\| \cdot\|Y\|$.

Furthermore, we obtain the following integral form of (1.3) (only consider real-valued functions).

Theorem 2 Let $\mu$ be a positive measure such that $\mu(\Omega)=1, f$ and $g$ be real-valued functions in $L^{2}(\mu)$, and let

$$
f \otimes_{A} g(x)=f(x) \cdot g(x)-f(x) \cdot \int_{\Omega} g d \mu-g(x) \cdot \int_{\Omega} f d \mu,
$$

then

$$
\left(\int_{\Omega}\left|f \otimes_{A} g\right| d \mu\right)^{2} \leq\left(\int_{\Omega} f^{2} d \mu\right)\left(\int_{\Omega} g^{2} d \mu\right)
$$

Equality holds if $g(x)=\left(f(x)-2 \int_{\Omega} f d \mu\right) \lambda$, where $\lambda$ is a non-negative constant. 


\section{The proofs of the theorems}

Proof of Theorem 1 Using the basic properties of the norm of a unitary space, we get

$$
\begin{aligned}
\sum_{i=1}^{n}\left\|y_{i}-2 b\right\|^{2} & =\sum_{i=1}^{n}\left\langle y_{i}-2 b, y_{i}-2 b\right\rangle \\
& =\sum_{i=1}^{n}\left(4\|b\|^{2}-2\left\langle y_{i}, b\right\rangle-2\left\langle b, y_{i}\right\rangle+\left\|y_{i}\right\|^{2}\right) \\
& =4 n\|b\|^{2}-2\left\langle\sum_{i=1}^{n} y_{i}, b\right\rangle-2\left\langle b, \sum_{i=1}^{n} y_{i}\right\rangle+\sum_{i=1}^{n}\left\|y_{i}\right\|^{2} \\
& =4 n\|b\|^{2}-2 n\|b\|^{2}-2 n\|b\|^{2}+\sum_{i=1}^{n}\left\|y_{i}\right\|^{2} \\
& =\sum_{i=1}^{n}\left\|y_{i}\right\|^{2} .
\end{aligned}
$$

By (2.1), using the Cauchy-Schwarz inequality (1.1) and the discrete form of the CauchySchwarz inequality, it follows that

$$
\begin{aligned}
\sum_{i=1}^{n}\left|\left\langle x_{i}, y_{i}-2 b\right\rangle\right| & \leq \sum_{i=1}^{n}\left\|x_{i}\right\| \cdot\left\|y_{i}-2 b\right\| \\
& \leq\left(\sum_{i=1}^{n}\left\|x_{i}\right\|^{2}\right)^{\frac{1}{2}}\left(\sum_{i=1}^{n}\left\|y_{i}-2 b\right\|^{2}\right)^{\frac{1}{2}} \\
& =\left(\sum_{i=1}^{n}\left\|x_{i}\right\|^{2}\right)^{\frac{1}{2}}\left(\sum_{i=1}^{n}\left\|y_{i}\right\|^{2}\right)^{\frac{1}{2}} .
\end{aligned}
$$

Similarly to (2.2), we have

$$
\sum_{i=1}^{n}\left|\left\langle x_{i}-2 a, y_{i}\right\rangle\right| \leq\left(\sum_{i=1}^{n}\left\|x_{i}\right\|^{2}\right)^{\frac{1}{2}}\left(\sum_{i=1}^{n}\left\|y_{i}\right\|^{2}\right)^{\frac{1}{2}}
$$

Combining (2.2) and (2.3), we infer that

$$
\begin{aligned}
\sum_{i=1}^{n}\left|x_{i} \otimes_{A} y_{i}\right| & =\frac{1}{2} \sum_{i=1}^{n}\left|2\left\langle x_{i}, y_{i}\right\rangle-\left\langle x_{i}, 2 b\right\rangle-\left\langle 2 a, y_{i}\right\rangle\right| \\
& =\frac{1}{2} \sum_{i=1}^{n}\left|\left\langle x_{i}, y_{i}-2 b\right\rangle+\left\langle x_{i}-2 a, y_{i}\right\rangle\right| \\
& \leq \frac{1}{2} \sum_{i=1}^{n}\left|\left\langle x_{i}, y_{i}-2 b\right\rangle\right|+\frac{1}{2} \sum_{i=1}^{n}\left|\left\langle x_{i}-2 a, y_{i}\right\rangle\right| \\
& \leq\left(\sum_{i=1}^{n}\left\|x_{i}\right\|^{2}\right)^{\frac{1}{2}}\left(\sum_{i=1}^{n}\left\|y_{i}\right\|^{2}\right)^{\frac{1}{2}}
\end{aligned}
$$

This is the inequality (1.3), as desired. 
Proof of Theorem 2 We first prove the following inequality:

$$
\int_{\Omega}\left|f\left(g-2 \int_{\Omega} g d \mu\right)\right| d \mu \leq\left(\int_{\Omega} f^{2} d \mu\right)^{\frac{1}{2}}\left(\int_{\Omega} g^{2} d \mu\right)^{\frac{1}{2}} .
$$

In fact, by the Cauchy-Schwarz inequality (1.2), we obtain

$$
\begin{aligned}
\int_{\Omega}\left|f\left(g-2 \int_{\Omega} g d \mu\right)\right| d \mu \\
\leq\left(\int_{\Omega} f^{2} d \mu\right)^{\frac{1}{2}}\left(\int_{\Omega}\left(g-2 \int_{\Omega} g d \mu\right)^{2} d \mu\right)^{\frac{1}{2}} \\
=\left(\int_{\Omega} f^{2} d \mu\right)^{\frac{1}{2}} \cdot\left(\int_{\Omega}\left(4\left(\int_{\Omega} g d \mu\right)^{2}-4\left(\int_{\Omega} g d \mu\right) \cdot g+g^{2}\right) d \mu\right)^{\frac{1}{2}} \\
=\left(\int_{\Omega} f^{2} d \mu\right)^{\frac{1}{2}} \cdot\left(4\left(\int_{\Omega} g d \mu\right)^{2} \mu(\Omega)-4\left(\int_{\Omega} g d \mu\right)^{2}+\int_{\Omega} g^{2} d \mu\right)^{\frac{1}{2}} \\
=\left(\int_{\Omega} f^{2} d \mu\right)^{\frac{1}{2}}\left(\int_{\Omega} g^{2} d \mu\right)^{\frac{1}{2}} .
\end{aligned}
$$

This is the inequality (2.4).

Similarly, we have

$$
\int_{\Omega}\left|g\left(f-2 \int_{\Omega} f d \mu\right)\right| d \mu \leq\left(\int_{\Omega} f^{2} d \mu\right)^{\frac{1}{2}}\left(\int_{\Omega} g^{2} d \mu\right)^{\frac{1}{2}} .
$$

From (2.4) and (2.5), we find that

$$
\begin{aligned}
& \int_{\Omega}\left|f \otimes_{A} g\right| d \mu \\
& \quad=\frac{1}{2} \int_{\Omega}\left|\left(g-2 \int_{\Omega} g d \mu\right) f+\left(f-2 \int_{\Omega} f d \mu\right) g\right| d \mu \\
& \quad \leq \frac{1}{2}\left(\int_{\Omega}\left|f\left(g-2 \int_{\Omega} g d \mu\right)\right| d \mu+\int_{\Omega}\left|g\left(f-2 \int_{\Omega} f d \mu\right)\right| d \mu\right) \\
& \quad \leq\left(\int_{\Omega} f^{2} d \mu\right)^{\frac{1}{2}}\left(\int_{\Omega} g^{2} d \mu\right)^{\frac{1}{2}} .
\end{aligned}
$$

The inequality (1.4) follows.

\section{An application}

Let $\left(a_{1}, b_{1}\right), \ldots,\left(a_{n}, b_{n}\right)$ be $n$ items of bivariate real data, $x=\left\{a_{1}, \ldots, a_{n}\right\}$ and $y=\left\{b_{1}, \ldots, b_{n}\right\}$, then their covariance $\operatorname{Cov}(x, y)$ is defined as [11]

$$
\operatorname{Cov}(x, y)=\frac{1}{n} \sum_{i=1}^{n}\left(a_{i}-a\right)\left(b_{i}-b\right)
$$

where $a=\frac{a_{1}+\cdots+a_{n}}{n}$ and $b=\frac{b_{1}+\cdots+b_{n}}{n}$. 
For the covariance $\operatorname{Cov}(x, y)$, it is well known that Pearson's product moment inequality is

$$
|\operatorname{Cov}(x, y)| \leq \operatorname{SD}(x) \cdot \operatorname{SD}(y)
$$

where $\operatorname{SD}(x)=\sqrt{\frac{1}{n} \sum_{i=1}^{n}\left(a_{i}-a\right)^{2}}$ and $\operatorname{SD}(y)=\sqrt{\frac{1}{n} \sum_{i=1}^{n}\left(b_{i}-b\right)^{2}}$.

Similarly, now we define this covariance of two $n$-tuples $X=\left(x_{1}, x_{2}, \ldots, x_{n}\right)$ and $Y=$ $\left(y_{1}, y_{2}, \ldots, y_{n}\right)$ of the unitary space $\mathbb{H}$ as

$$
\operatorname{Cov}(X, Y)=\frac{1}{n} \sum_{i=1}^{n}\left\langle x_{i}-a, y_{i}-b\right\rangle,
$$

where $a=\frac{x_{1}+\cdots+x_{n}}{n}$ and $b=\frac{y_{1}+\cdots+y_{n}}{n}$.

Set $\alpha_{i}=x_{i}-a$ and $\beta_{i}=y_{i}-b, i=1, \ldots, n$. Note that

$$
\begin{aligned}
x_{i} \otimes_{A} y_{i} & =\left\langle\alpha_{i}+a, \beta_{i}+b\right\rangle-\left\langle\alpha_{i}+a, b\right\rangle-\left\langle a, \beta_{i}+b\right\rangle \\
& =\left\langle\alpha_{i}, \beta_{i}\right\rangle-\langle a, b\rangle \\
& =\left\langle x_{i}-a, y_{i}-b\right\rangle-\langle a, b\rangle .
\end{aligned}
$$

Hence, (1.3) can be written in the following form:

$$
\sum_{i=1}^{n}\left|\left\langle x_{i}-a, y_{i}-b\right\rangle-\langle a, b\rangle\right| \leq\|X\| \cdot\|Y\|,
$$

where $\|X\|=\left(\sum_{i=1}^{n}\left\|x_{i}\right\|^{2}\right)^{1 / 2}$ and $\|Y\|=\left(\sum_{i=1}^{n}\left\|y_{i}\right\|^{2}\right)^{1 / 2}$.

Using the triangle inequality on the left side of (3.1), we obtain

$$
|n \operatorname{Cov}(X, Y)-n\langle a, b\rangle| \leq\|X\| \cdot\|Y\| \text {. }
$$

Finally, we can simply state the above result, as follows.

Theorem 3 Let $X=\left(x_{1}, x_{2}, \ldots, x_{n}\right)$ and $Y=\left(y_{1}, y_{2}, \ldots, y_{n}\right)$ be two n-tuples of the unitary space $\mathbb{H}$, then

$$
|\operatorname{Cov}(X, Y)-\langle a, b\rangle| \leq \frac{1}{n}\|X\| \cdot\|Y\| .
$$

\section{Competing interests}

The author declares to have no competing interests.

\section{Acknowledgements}

This work was partially supported by the National Natural Science Foundation of China (Grant No. 11171053).

Received: 10 December 2014 Accepted: 28 May 2015 Published online: 17 June 2015

\section{References}

1. Dragomir, SS: A survey on the Cauchy-Buniakowsky-Schwarz type discrete inequalities. J. Inequal. Pure Appl. Math. 4, Article 63 (2003)

2. Ackermann, N: A Cauchy-Schwarz type inequality for bilinear integrals on positive measures. Proc. Am. Math. Soc $133,2647-2656(2005)$ 
3. Alzer, H: A refinement of the Cauchy-Schwarz inequality. J. Math. Anal. Appl. 168, 596-604 (1992)

4. Alzer, H: On the Cauchy-Schwarz inequality. J. Math. Anal. Appl. 234, 6-14 (1999)

5. Carbery, A: A multilinear generalization of the Cauchy-Schwarz inequality. Proc. Am. Math. Soc. 132, 3141-3152 (2004)

6. Dragomir, SS: On the Cauchy-Buniakowsky-Schwarz inequality for sequences in inner product space. Math. Inequal. Appl. 3, 385-398 (2000)

7. Masjed-Jamei, M: A functional generalization of the Cauchy-Schwarz inequality and some subclasses. Appl. Math. Lett. 22, 1335-1339 (2009)

8. Mitrinović, DS, Pećarić, JE, Fink, AM: Classical and New Inequalities in Analysis. Kluwer Academic, Dordrecht (1993)

9. Wada, S: On some refinement of the Cauchy-Schwarz inequality. Linear Algebra Appl. 420(2-3), 433-440 (2007)

10. Rudin, W: Real and Complex Analysis. McGraw-Hill, New York (1987)

11. Fisher, RA: Statistical Methods, Experimental Design, and Scientific Inference. Oxford University Press, New York (1990)

Submit your manuscript to a SpringerOpen ${ }^{\circ}$ journal and benefit from:

- Convenient online submission

Rigorous peer review

- Immediate publication on acceptance

- Open access: articles freely available online

- High visibility within the field

- Retaining the copyright to your article 